\title{
Mechanisms of creep deformation in polycrystalline Ni-base disk superalloys
}

\author{
R.R. Unocic ${ }^{\text {a,* }}$, G.B. Viswanathan ${ }^{\text {a, }}$ P.M. Sarosi ${ }^{\text {a }}$, \\ S. Karthikeyan ${ }^{\text {b }}$, J. Li ${ }^{\text {a }}$, M.J. Mills ${ }^{\text {a }}$ \\ ${ }^{a}$ Department of Materials Science and Engineering, The Ohio State University, 477 Watts Hall, 2041 College Road, Columbus, OH 43210, USA \\ ${ }^{\mathrm{b}}$ Department of Materials Engineering, Indian Institute of Science Bangalore, Karnataka 560012, India
}

Received 6 June 2006; received in revised form 16 August 2006; accepted 16 August 2006

\begin{abstract}
This paper reviews the presently proposed mechanisms for creep of $\gamma^{\prime}$ strengthened Ni-base superalloys that are typically used for disk applications. Distinct creep strength controlling modes, such as dislocation-coupled antiphase-boundary shearing, shearing configurations involving superlattice stacking faults, Orowan looping, climb by-pass, and microtwinning have been observed. These are strongly influenced by the scale of the $\gamma^{\prime}$ precipitating phase and are operative within specific ranges of temperature and stress. Insight from more recent experimental findings concerning microtwinning and extending stacking fault mechanisms suggest important similarities between these deformation modes. It is suggested that local atomic reordering in the wake of Shockley partials is responsible for the temperature dependence exhibited in this regime.
\end{abstract}

(C) 2007 Elsevier B.V. All rights reserved.

Keywords: Creep; Deformation mechanisms; Ni-base superalloy; Microtwinning

\section{Introduction}

Ni-base superalloys are used extensively in the hot section of gas turbine engines because of their inherent elevated temperature strength and creep resistance. As such, aircraft engine manufactures are continually striving to push the envelope of the capabilities of such high temperature structural materials in order to increase both engine performance and efficiency.

While extensive experimentation has been performed on superalloys as part of engine certification programs, there has been comparatively little investment in understanding the fundamental deformation mechanisms. In particular, the connection between microstructure scale - and hence heat treatment and deformation process has not been fully elucidated. In this paper, the focus will be on the mechanisms that are operative at the upper bound of typical service temperatures. In terms of creep properties, it is these time-dependent deformation mechanisms that may be performance limiting. Therefore, they must be more fully understood in order to rationally design alloys and microstructures with improved creep performance, and to

\footnotetext{
* Corresponding author.

E-mail address: unocic.4@osu.edu (R.R. Unocic).
}

understand the degradation of properties as a function of elevated temperature exposure.

The creep deformation behavior of two polycrystalline $\mathrm{Ni}$ based superalloys used for disk applications - René 88DT and René 104 - have been investigated. These alloys attain a significant portion of elevated temperature strength and creep resistance from the presence of coherent, ordered, intermetallic $\gamma^{\prime}\left(\mathrm{Ni}_{3} \mathrm{Al}\right)$ precipitates that effectively impede dislocation motion at elevated temperatures. The previous investigation and models proposed for shearing of the $\gamma^{\prime}$ precipitates will be briefly reviewed, and recent work will then be described in which a combination of transmission electron microscopy (TEM) characterization techniques have been used to thoroughly identify deformation structures that form during creep and to provide a mechanistic basis for the development of sound, physically based models for creep in these disk alloys.

\section{Previous analysis of deformation mechanisms in disk alloys}

A number of models have been proposed in the literature which describe the shearing of $\gamma^{\prime}$ precipitates via $1 / 2\langle 110\rangle$ matrix dislocations, $1 / 6\left\langle\begin{array}{lll}1 & 12\rangle & \text { Shockley partials, } 1 / 3\langle 112\rangle\end{array}\right.$ 
superlattice Shockley partials, and the corresponding formation of antiphase boundaries (APBs), complex stacking faults (CSFs), as well as superlattice intrinsic (SISFs) and superlattice extrinsic (SESFs) stacking faults.

\subsection{Kear mechanism}

Kear et al. [1] performed detailed TEM analysis of low misfit alloy single crystals, enabling them to perform detailed TEM investigation of slip-plane sections following creep deformation. They observed relatively planar dislocation configurations on $\left\{\begin{array}{lll}1 & 1 & 1\end{array}\right\}$ planes in which the large strengthening $\gamma^{\prime}$ precipitates are sheared, leaving behind faults, while the matrix remains unsheared. It should be noted that it is unclear whether the alloys studied by Kear et al. contained fine $\gamma^{\prime}$ precipitates. The observed shearing process spawned the model illustrated in Fig. 1. In the descriptions to follow, we utilize the Thompson tetrahedron notation illustrated in Fig. 1 inset indicating the various Burgers vectors associated with the $\alpha\left\{\begin{array}{ll}\overline{1} & 1 \\ 1\end{array}\right\}$ plane [2]. The process of shearing the larger $\gamma^{\prime}$ particles is presumed to occur by the cooperative movement of coupled $a / 2\left[\begin{array}{lll}1 & 1 & 2\end{array}\right]$ dislocations (3D $\left.\alpha\right)$, each of which is dissociated into three $a / 6\left[\begin{array}{ll}1 & 1\end{array}\right]$ partials $(D \alpha)$. Their TEM analysis indicated that all partials are of the same type. The individual $\mathrm{D} \alpha$ partials are not shown in the figure.

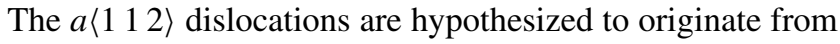
the interaction of two different $a\langle 101\rangle$ superdislocations originating from different slip systems. For example:

$a\left[\begin{array}{lll}0 & 1 & 1\end{array}\right]+a\left[\begin{array}{lll}1 & 0 & 1\end{array}\right]=a\left[\begin{array}{lll}1 & 1 & 2\end{array}\right]$

Clearly, this model then requires a high symmetry orientation such that two slip systems experience a relatively large shear stress. The existence of $a\left[\begin{array}{ll}1 & 1\end{array} 2\right]$ dislocations in the matrix was not reported or proven. In light of discussion to follow, specifically regarding the operation of microtwinning, it should be noted that the sequence of partial dislocations is identical to that which has now been shown to be associated with the microtwinning process. The primary difference is that in the Kear mechanism the Shockley partials comprising each $a / 2\left[\begin{array}{ll}1 & 1\end{array} 2\right]$ dislocation are assumed to be on a single glide plane while they are on adjacent $\left\{\begin{array}{lll}1 & 1 & 1\end{array}\right\}$ planes for the microtwinning mechanism.

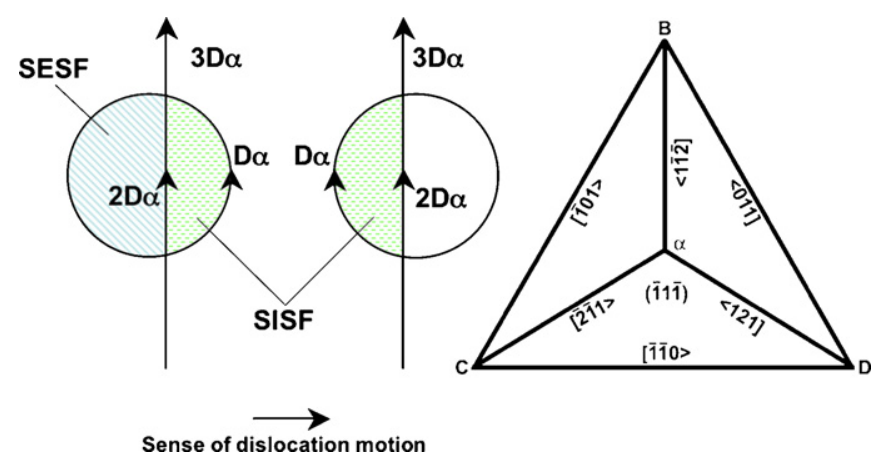

Fig. 1. Proposed mechanism by Kear et al. [1] of $\gamma^{\prime}$ precipitate shear by coupled Shockley partials creating SISF/SESF pairs (Inset: Thompson tetrahedron used in notation of Burgers vectors. After Hirth and Lothe [2]).

\subsection{Decamps mechanisms}

This series of models are based on the movement of isolated $a / 2\langle 110\rangle$ dislocations in the matrix. The early observations indicated the presence of superlattice extrinsic stacking faults (SESFs) in the large $\gamma^{\prime}$ precipitates, with linking $a / 2\langle 110\rangle$ dislocations in the matrix $[3,4]$. The process illustrated in Fig. 2A (Decamps Model A) was postulated to explain these observations, involving first the shearing of the precipitates by the matrix dislocation (DC), forming an APB in the precipitate. Due to the relatively high energy of this fault, these authors propose that it is energetically favorable to nucleate a Shockley partial $(\alpha \mathrm{B})$ on the $\left\{\begin{array}{lll}1 & 1 & 1\end{array}\right\}$ plane adjacent to the APB. The composite partial, combining DC and $\alpha \mathrm{B}$ is equivalent to a $1 / 3\langle 112\rangle$ partial (2D $\alpha)$ which is spread on two $\left\{\begin{array}{llll}1 & 1 & 1\end{array}\right\}$ planes. The nucleation of the Shockley partial is presumed to convert the high energy APB into a lower energy SESF. Presumably, if the Shockley partial were to nucleate in the plane of the APB, a low energy SISF could also be formed.

A principal concern with this model is that the incorrect nearest neighbors associated with the APB will not be affected by the nucleation of a partial on the plane above the APB. Hence, additional, unspecified atomic rearrangements would be required to create the SESF.

In more recent publications, Decamps et al. [5] instead propose that these same shearing processes are initiated by a dissociated $a / 2\langle 110\rangle$ matrix dislocation $(\mathrm{DC} \rightarrow \mathrm{D} \alpha+\alpha \mathrm{C})$. This process is illustrated in Fig. 2B (Decamps Model B). As the leading $\mathrm{D} \alpha$ partial enters the secondary $\gamma^{\prime}$ precipitate, a complex stacking fault (CSF) is created. Since this fault has a very large energy (larger than that of an APB), there exists a driving force to lower its energy. The authors propose that this driving force is relieved by the nucleation of another D $\alpha$ Shockley partial on the $\left\{\begin{array}{lll}1 & 1 & 1\end{array}\right\}$ plane above the CSF. The resultant composite partial, comprised of two $\mathrm{D} \alpha$ Shockley partials on adjacent $\left\{\begin{array}{lll}1 & 1 & 1\end{array}\right\}$ planes, is therefore equivalent to an $a / 3\langle 112\rangle$ partial ( $2 D \alpha)$, and the fault trailing through the $\gamma^{\prime}$ precipitate is presumed to be an SESF. As will become clear through our discussion to follow, this presumption is incorrect, as in fact the process described by Decamps et al. [5] would instead create a two-layer CSF. Additional atomic rearrangements are required in order to generate a low energy SESF.

In a third variant on their models (not reproduced here), Decamps et al. [6] have also suggested that if the two partials of the parent DA matrix dislocation cross-over each other upon entering the particle (i.e. the $\alpha \mathrm{C}$ partial enters first), an extrinsic CSF would be created. Nucleation of the D $\alpha$ Shockley partial on the fault plane would convert it to an SISF. Once again, the atomic rearrangements required for each of these steps are not fully specified.

\subsection{Chen and Knowles mechanism}

Knowles and Chen [7] report that SESF configurations in the larger $\gamma^{\prime}$ particles coexist with microtwinning for particular crystal orientations in CMSX-4, a single crystal alloy with relatively large lattice misfit. The initiation and propagation of the 


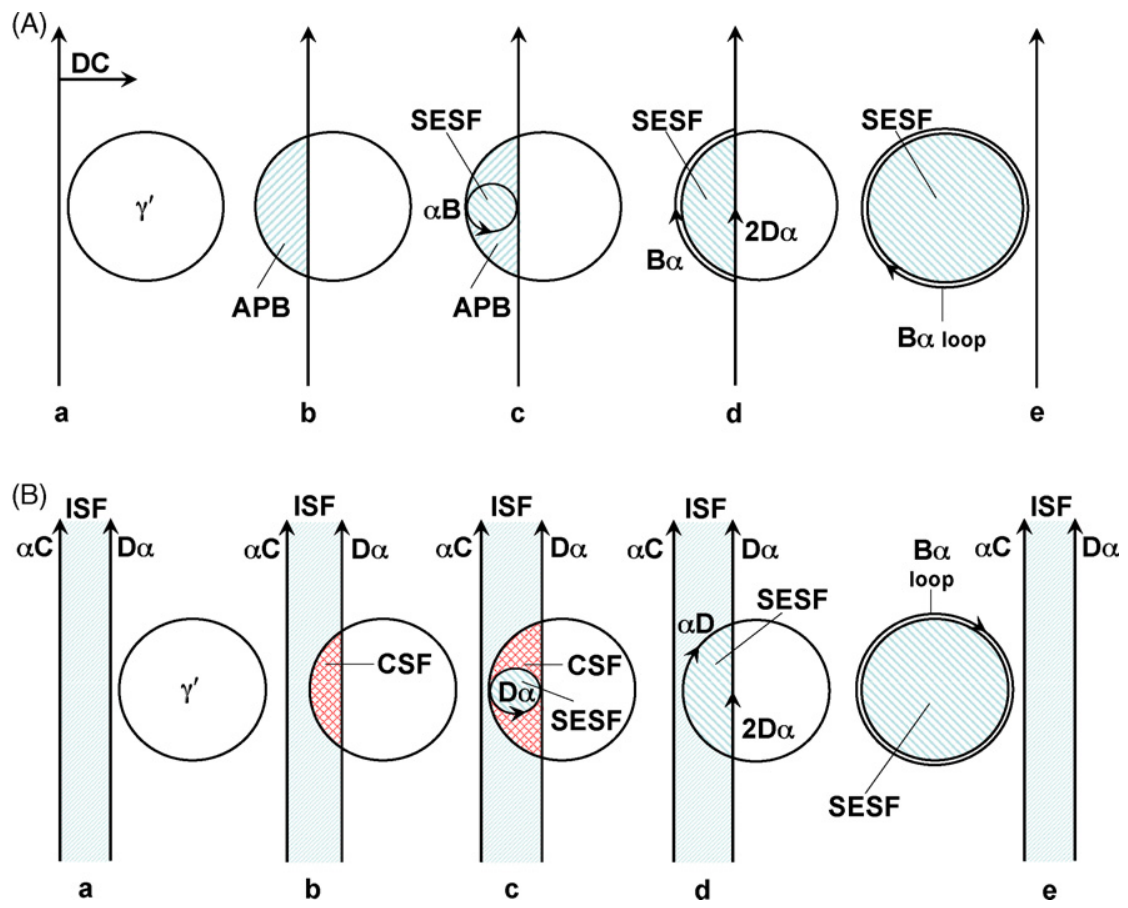

Fig. 2. (A) Schematic of $\gamma^{\prime}$ precipitate shear mechanism and SESF formation as proposed by Decamps and co-workers [3,4] to model $\gamma^{\prime}$ precipitate shear by (a) a single matrix dislocation $a / 2\left\langle\begin{array}{lll}1 & 1 & 0\rangle\end{array}\right.$; (b) APB formation; (c) nucleation of Shockley partial on adjacent $\left\{\begin{array}{llll}1 & 1 & 1\end{array}\right\}$ plane; (d) removal of APB; (e) creation of SESF within the precipitate. Decamps Model A [3,4]. (B) Proposed mechanism of $\gamma^{\prime}$ precipitate shear and SESF formation by (a) a dissociated $a / 2\langle 110\rangle$ matrix dislocation with (b) leading D $\alpha$ shearing the $\gamma^{\prime}$ and creating a CSF; (c) nucleation of Shockley partial on adjacent $\left\{\begin{array}{lll}1 & 1 & 1\end{array}\right\}$ plane; (d) creation of SESF; (e) looping by B $\alpha$ Shockley partial after shearing commences. Decamps Model B [5].

microtwins, as well as the formation of isolated SESFs in the $\gamma^{\prime}$ particles, are presumed to be associated with the operation of $a / 3\langle 112\rangle$ partials (e.g. $2 \mathrm{D} \alpha$ ) gliding on every second $\left\{\begin{array}{lll}1 & 1 & 1\end{array}\right\}$ plane, as illustrated in Fig. 3. However, this net displacement is considered to be due to the passage of two different $a / 3\langle 112\rangle$ partials on every $\{1111\}$ plane (e.g. $2 \alpha B$ then $2 \alpha C$ ). Passage of the first net 2D $\alpha$ will create an SESF, while subsequent passages will thicken the twin. For an opposite sense of applied shear stress, an SISF would be expected. The authors make the very interesting observation that microtwins are observed only in orientations for which SESFs are observed as well.

The authors propose that the $a / 3\langle 112\rangle$ twinning partials arise from a pole mechanism, which originate with the interaction of

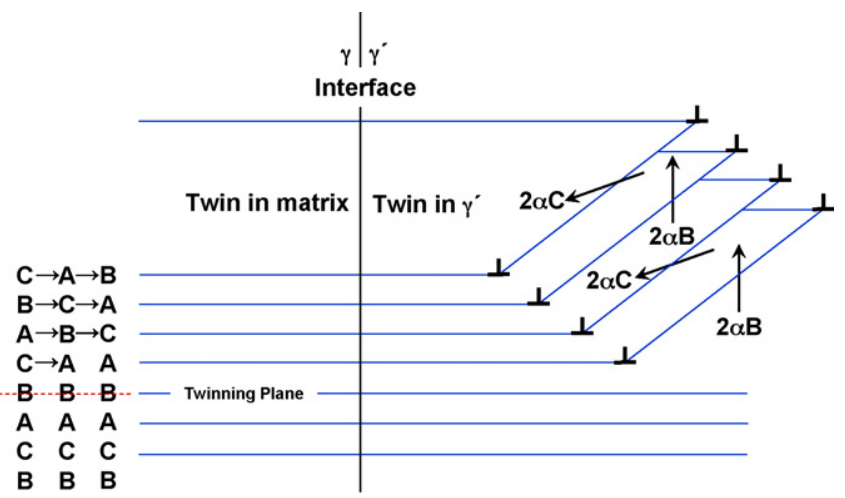

Fig. 3. Model of twin formation in $\gamma / \gamma^{\prime}$ superalloys by the passage of two different $a / 3\left\langle\begin{array}{lll}1 & 1 & 2\rangle\end{array}\right)$ super Shockley pairs $(2 \alpha \mathrm{B}$ and $2 \alpha \mathrm{C})$ on adjacent $\left\{\begin{array}{llll}1 & 1 & 1\end{array}\right\}$ planes. After Knowles and Chen [7]. two identical $a / 2\langle 110\rangle$ matrix dislocations (DC). The temperature and stress dependence of the process is associated with the critical size for nucleation of the twin from the pole. Presumably, the subsequent propagation of the twinning partials is rapid and not rate controlling. A difficulty with this model for twinning is that it requires successive shear of $\left\{\begin{array}{lll}1 & 1 & 1\end{array}\right\}$ planes in directions that are necessarily of small Schmid factor (presuming that the net shear direction has large Schmid factor). The model considers only the possibility of conservative shearing of the $\mathrm{L}_{2}$ lattice. As will now be discussed, Kolbe [8] has proposed an entirely different scenario for the twinning process.

\subsection{Kolbe mechanism for microtwinning}

Kolbe et al. [9] performed in situ TEM deformation experiments at elevated temperature which indicated a distinct transition in deformation mechanism with temperature. Below about $760^{\circ} \mathrm{C}$, dislocation motion is observed to be jerky, in which encounters with $\gamma^{\prime}$ precipitates resulted in distinct waiting times. The shearing dislocations were reported to be $a / 2\langle 110\rangle$ type, although no direct evidence is provided to support this claim. In situ deformation at higher temperature gives rise to a distinctly different mode of shearing in which extended faults propagate continuously and viscously through both particles and matrix. These extended faults are associated with partials that move in a correlated manner as pairs. Kolbe deduced that these partials may be $a / 6\langle 112\rangle$ partials of the same Burgers vector (e.g. a pair of $\mathrm{D} \alpha$ partials), and that they may be traveling on parallel $\left\{\begin{array}{lll}1 & 1 & 1\end{array}\right\}$ planes, as illustrated in Fig. 4. Without detailed 


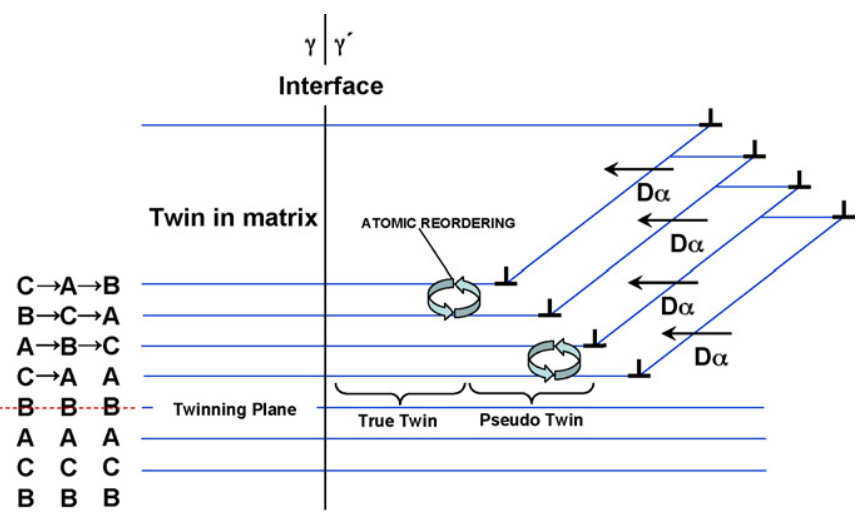

Fig. 4. Schematic representation of microtwinning mechanism from shear by identical Shockley partials $(\mathrm{D} \alpha)$ transcending both the $\gamma$ matrix and $\gamma^{\prime}$ precipitate on adjacent $\left\{\begin{array}{lll}1 & 1 & 1\end{array}\right\}$ planes which then require atomic reordering in $\gamma^{\prime}$ to convert stacks of CSF (created by D $\alpha$ ) into a true twinned structure. After Kolbe [8].

confirmation of this hypothesis, Kolbe further deduced that these were in fact microtwins, and that the temperature dependence of the process may be associated with reordering that would ensue

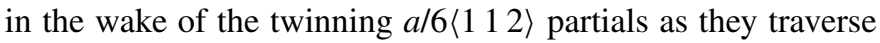
the $\gamma^{\prime}$ particles.

This remarkable deduction, based on rather limited experimental observation, has now been validated based on more recent, detailed TEM analysis of post-mortem specimens, as will be discussed in the next section. This work provides the physical insight upon which a quantitative model of microtwinning has been recently developed. The essential elements of this model are summarized in Section 3.2.

\section{Detailed analysis and model of microtwinning}

\subsection{Attributes of microtwinning}

Viswanathan et al. [10] and Sarosi et al. [11] have recently presented a more complete TEM analysis of the microtwinning process using diffraction contrast and high-resolution TEM (HRTEM) investigation. This work was initially performed on René 88DT deformed in creep at $834 \mathrm{MPa}$ and $650^{\circ} \mathrm{C}$. Very similar structures have also been observed in the alloy René 104 deformed at $690 \mathrm{MPa}$ and $677^{\circ} \mathrm{C}$. HRTEM observations in René 88DT showed conclusively that thicker microtwins (of the order of several $\left\{\begin{array}{lll}1 & 1 & 1\end{array}\right\}$ interplanar spacings or greater in thickness) have the $\mathrm{L}_{2}$ structure in a true twin relationship with the matrix. An example of microtwins traversing a grain in René 104 is shown in Fig. 5, with twin reflections in inset diffraction pattern. Detailed diffraction contrast image analyses have demonstrated that the twinning partials are in fact identical $1 / 6\langle 112\rangle$ partials. This analysis has been conducted on samples deformed to small strain levels $(0.2 \%$ plastic strain). Under these conditions, a number of twins had not traversed entire grains, and the leading partials of the twin could therefore be analyzed. Contrast analysis has indicated that the leading partials are closely coupled $a / 6\left\langle\begin{array}{lll}1 & 1 & 2\rangle\end{array}\right.$ partials. In the wake of these partials is an extrinsic stacking fault extending through both precipitates and matrix. The next (third) partial shears the matrix regions, but is unable

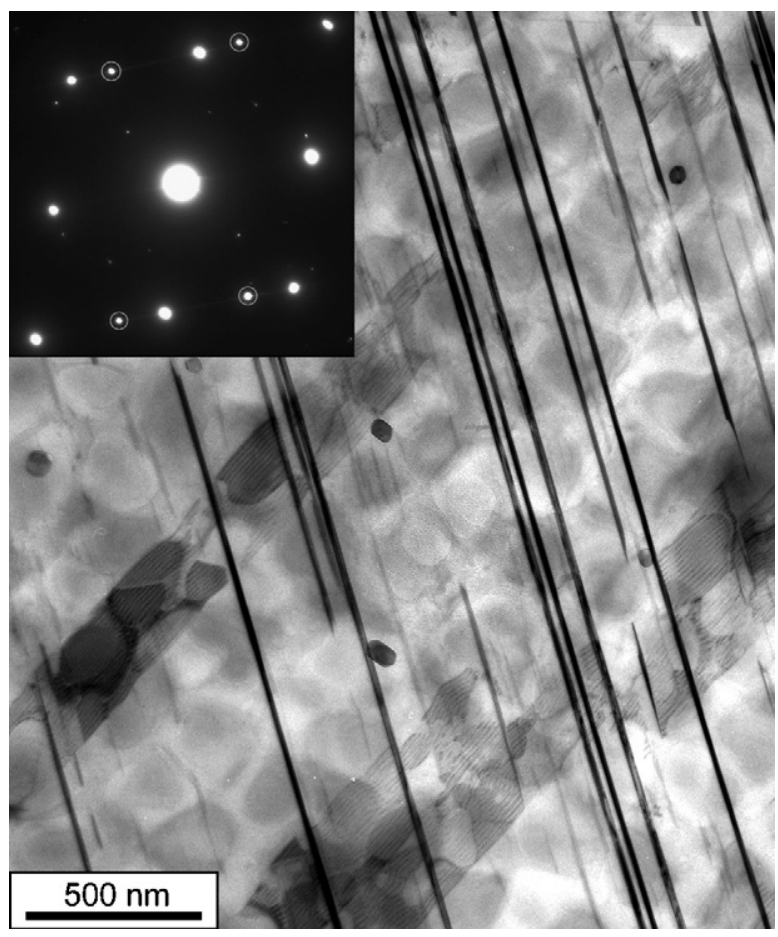

Fig. 5. Microtwinning deformation mode operative in creep deformed René 104 at $690 \mathrm{MPa}$ and $677^{\circ} \mathrm{C}$ (Inset SADP showing presence of twin reflections, circled).

to shear the larger secondary particles. Consequently, the third partial is also deposited around the secondary particles. In the wake of the next (fourth) partial, the matrix and particles are once again sheared continuously. Analysis of thicker twins indicates that the twinning partials are often, but not always paired, as discussed initially by Kolbe [8].

These results are fully consistent with the reordering hypothesis of Kolbe [8], as shown in Fig. 4. In order for a true twin structure to originate from the sequential passage of $a / 6\langle 112\rangle$ partials, atomic reordering must be occurring in the wake of the partials. Otherwise, a pseudotwin would be created with a large number of unfavorable near-neighbor atomic sites since the structure would essentially be a stacking of CSFs. Assuming that reordering occurs very rapidly relative to the movement of the partials, then an SESF would be created in the wake of the first two partials. However, the third partial is unable on its own to shear the larger secondary particles since this would once again create a third layer of the twin with a large number of incorrect nearest neighbors. When the fourth partial approaches the secondary particles that have been looped by the third partial, these two partials are able to cooperatively shear the secondary particles via exchange of atoms on the adjacent (1 111 ) planes, in a manner similar to the reordering in the wake of the first two partials.

The diffraction contrast analyses described above are challenging due to (a) the close separation between the partials (particularly the first two), (b) the complicating effect of the $\gamma^{\prime}$ particles, and the differential thinning of matrix and particles, and (c) the obscuring effect of the faults adjacent to the twinning partials. Additional support for the Kolbe reordering process has 
recently been obtained via HRTEM analysis of a two-layer fault configuration, as is formed following the first two twinning partials [11]. Comparison of the (100) superlattice contrast with simulated images for several different shearing scenarios has been performed. The analysis indicates that the thin, two-layer fault is not consistent with simulations for a perfect SESF as would be created by the movement of $a / 3\left\langle\begin{array}{lll}1 & 1 & 2\rangle\end{array}\right.$ partials on adjacent planes. Rather, the two-layer fault is more closely related to a two-layer CSF, albeit with an apparently reduced degree of order in the two layers. Such a structure can only have been created by movement of $a / 6\langle 112\rangle$ partials on adjacent planes, with partial reordering occurring in their wake. This result indicates that complete reordering to the $\mathrm{L}_{2}$ structure is not necessary to enable propagation of the leading twinning partials. Reordering must nevertheless continue as a function of time at temperature, as evidenced by the true twin structure of the thicker twins. It is noted that the detailed ordering state at the twin matrix interface has not yet been successfully investigated, and could provide additional insight into the spatial extent of the reordering process.

\subsection{Microtwinning model}

A quantitative model for microtwinning based on Shockley partial shearing process described above has been proposed by Karthikeyan et al. [12]. The model is based upon the assumption that the cooperative movement of the Shockley partial pairs can occur only when the energy of the two-layer fault in its wake is reduced via reordering. Since reordering is a time and temperature dependent process, the twinning partial velocity also has these attributes. The reordering process is assumed to operate within the larger secondary particles, while the tertiary particles are assumed to be small enough to shear athermally. These assumptions then set upper and lower bounds on the stress range over which the model will apply. Specifically, if $\tau_{\text {app }}$ is larger than $750 \mathrm{MPa}$, the secondary particles would be sheared athermally. On the other hand, if $\tau_{\text {app }}$ is smaller than $f_{3} \Gamma_{\mathrm{pt}} / b_{\mathrm{tp}}$, then the twinning partials would be unable to proceed through the field of tertiary particles (where $f_{3}$ is the volume fraction of tertiary $\gamma^{\prime}$ precipitates, $\Gamma_{\mathrm{pt}}$ is the pseudotwin energy, and $b_{\mathrm{tp}}$ is the magnitude of the Burgers vector of the twinning partials). Thus, the expected stress range under which microtwinning would be expected is 20-750 MPa. Given that the energy of the twolayered pseudotwin is $\Gamma_{\mathrm{pt}}$, and that of a two layer, true twin is $\Gamma_{\mathrm{tt}}$, Karthikeyan et al. [12] has proposed that the shear strain rate be given by

$\dot{\gamma}=\rho_{\mathrm{tp}} b_{\mathrm{tp}} v=\rho_{\mathrm{tp}} b_{\mathrm{tp}} \frac{D_{\mathrm{ord}}\left(b_{\mathrm{tp}} / x^{2}\right)}{\ln \left[f_{2} \Delta \Gamma /\left(2 \tau_{\mathrm{eff}} b_{\mathrm{tp}}-f_{2} \Gamma_{\mathrm{tt}}\right)\right]}$

where $\rho_{\text {tp }}$ is the density of mobile twinning partials, $D_{\text {ord }}$ is the diffusion coefficient for ordering, $x$ is the short range diffusion length (assumed to be several nearest neighbor distances, or $\sim 2 b$ ), and $f_{2}$ is the volume fraction of the secondary $\gamma^{\prime}$ precipitates. The form of Eq. (2) originates from the assumption of an exponential decay of $\Gamma$ with time [13]. The effective stress, $\tau_{\text {eff, }}$ in the presence of tertiary $\gamma^{\prime}$ precipitates, is given by

$\tau_{\mathrm{eff}}=\tau-\frac{f_{3} \Gamma_{\mathrm{pt}}}{2 b_{\mathrm{tp}}}$

The experimental values of parameters such as dislocation density $\rho_{\text {tp }}$, volume fraction of the secondary $\gamma^{\prime}$ precipitate that are critical to the prediction can be determined directly from TEM observations. The magnitude of the strain rate predicted by this model is in remarkably good agreement with the observed strain rate [12], particularly for the strain level at which the density of twinning partials is measured. While the level of agreement with experiment may be somewhat fortuitous, the basic kinetics associated with the twinning partial velocity appears to be reasonable.

Disk alloys in this temperature regime typically exhibit creep curves having a minimum rate, with a prolonged increase of creep rate with time. In the present model, such an increase would occur as a result of a decrease in $f_{3}$ with time (while $f_{2}$ increases, assuming a constant $\gamma^{\prime}$ total volume fraction). This Oswald ripening process has been observed experimentally under creep conditions [14]. In addition, the evolution of the twinning partial density as a function of strain has not yet been characterized, although the number of twins does increase with strain [10]. Finally, as the tertiary volume fraction decreases during thermal exposure, it is possible that the operation of $1 / 2\left[\begin{array}{lll}1 & 1 & 0\end{array}\right]$ matrix dislocations becomes increasingly important. This hypothesis is based on earlier observations [10] that in a coarse microstructure (small value of $f_{3}$ ) resulting from a slow cooling rate, deformation is dominated by $1 / 2\langle 110\rangle$ dislocation activity in the matrix, and SESF shearing in the secondary $\gamma^{\prime}$ precipitates.

\section{Additional deformation modes}

In addition to the observation of microtwinning, several additional deformation modes have been observed in a systematic study of René 88DT and René 104. These additional mechanisms are summarized below.

\subsection{Orowan bowing/APB shearing}

Shearing of precipitates by coupled $a / 2\langle 110\rangle$ pairs is clearly an important deformation mode at lower temperatures [15]. Consistent with this previous work, shearing involving the creation of APBs only appears to occur for high creep stresses and the lowest temperatures for which creep testing has been performed. In René 88DT at $972 \mathrm{MPa}$ and $650^{\circ} \mathrm{C}$, the manner of shearing is different from the strongly coupled mode observed at lower temperatures [10]. In this mode, the first $a / 2\langle 110\rangle$ dislocation shears the finest precipitates, but loops around the larger precipitates,

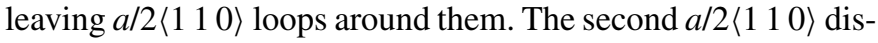
location, if it is on the same glide plane, then meets up with the loop, enabling the cooperative shearing of the precipitate while minimizing the width of the APB. It is noted that we have not directly observed this intermediate, shearing configuration, but infer this scenario from two salient features of the struc- 

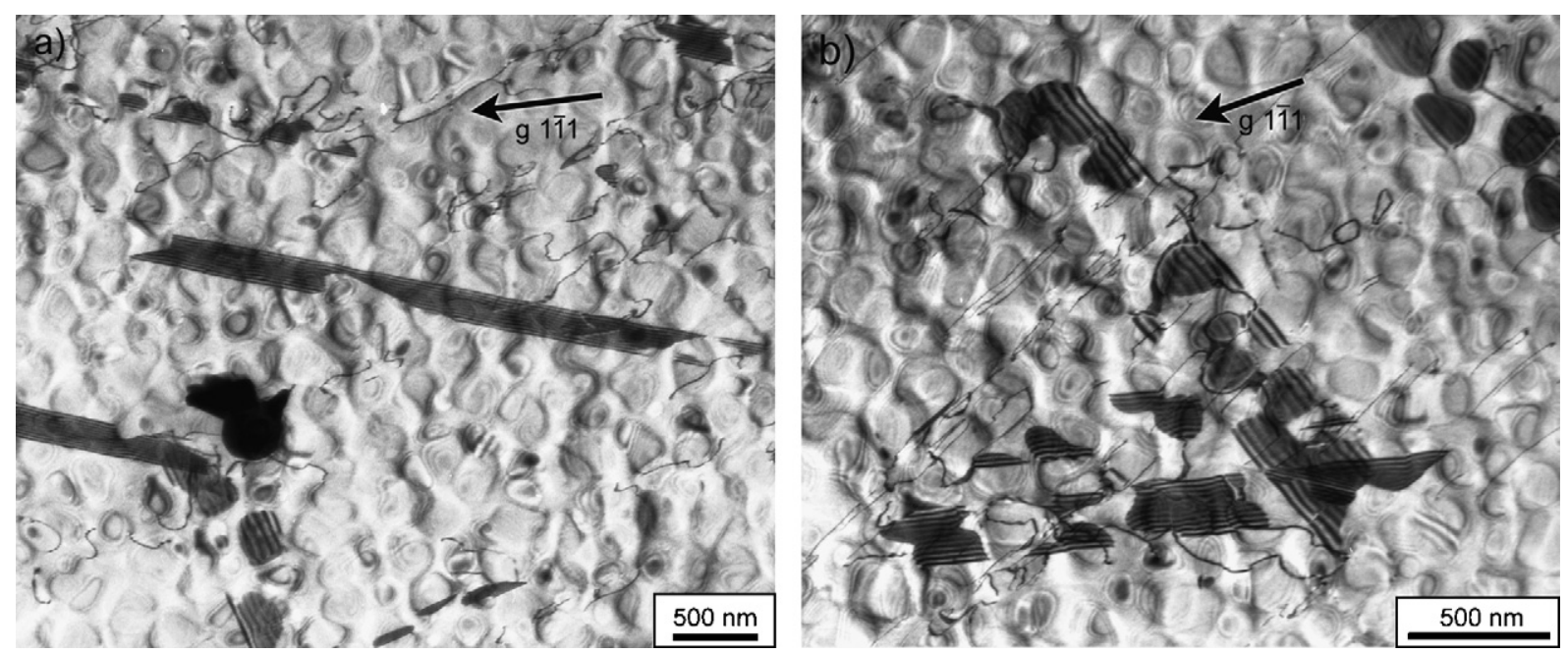

Fig. 6. Bright field TEM images of (a) continuous extended stacking faults and (b) discontinuous extended stacking faults alongside $a / 2\langle 110\rangle$ matrix dislocations in creep deformed René 104 under a tensile stress of $345 \mathrm{MPa}$ at $760^{\circ} \mathrm{C}(g=1 \overline{1} 1)$.

tures. First, the slip mode is very planar, as judged from edge-on views of the glide plane. Second, only singular loops are generally observed around a given secondary precipitate. Unless this APB shearing mechanism were operative, thereby annihilating the loops, intense strain hardening due to the presence of multiple loops around each particle would occur.

\subsection{Isolated faulting}

In René 88DT in the coarser microstructure, produced by slower cooling from supersolvus temperatures, the creep rates are much faster, and the time to $0.2 \%$ creep strain many times more rapid than in the fine microstructure. Recall that for the fine microstructure, microtwinning is the dominant process. However, in the coarse microstructure, $a / 2\langle 110\rangle$ dislocations are observed in the matrix, and extrinsic faults are created in the secondary precipitates. Thus, an $a / 6\left\langle\begin{array}{lll}1 & 1 & 2\rangle\end{array}\right.$ partial, or more precisely a composite partial $\mathrm{B} \alpha$ comprised of a $\mathrm{C} \alpha$ and a $\mathrm{D} \alpha$ on adjacent planes, surrounds each of the faulted precipitates. A possible explanation for these isolated faults is provided by the Decamps model of Fig. 2b. It is important to note that the movement of the 2D $\alpha$ through the secondary particles will require that reordering occur in order to avoid creation of a large number of incorrect nearest neighbors, and one that clearly provides a link to the microtwinning mechanism described above.

\subsection{Extended stacking faults}

At temperatures slightly higher than that for the microtwinning regime $\left(760^{\circ} \mathrm{C}\right)$, extended stacking faults are observed that either continuously traverse both matrix and precipitates, or can exhibit regions of isolated faulting within the precipitates for some of their length (Fig. 6a). These faults are however much shorter than the microtwin structures that generally span across entire grains. These extended faults are typically on the order of a few micrometers in width. Diffraction contrast analysis indicates that the faults are predominantly extrinsic in nature following tensile creep, although a significant density of intrinsic faults are also observed. These faults tend to co-exist with $a / 2\langle 110\rangle$ matrix dislocations, which often exhibit dissociation in the matrix (Fig. 6b).

A possible explanation for these faults is provided by an extension of the Decamps model of Fig. 2b, whereby the 2D $\alpha$ is able to sweep out beyond the secondary particles, and also encompasses the matrix. This will create an ESF in the matrix and an SESF in the precipitates, if reordering is allowed to occur. This mechanism has been proposed recently by Decamps et al. [5] to explain the presence of these extended faults, although they do not recognize the need for reordering to form SESFs in the particles. Thus, the leading $2 \mathrm{D} \alpha$ partial has a configuration identical to that envisaged for the leading partial pairs of the microtwin.

The insight that reordering is occurring in this case as well has another important implication on the mobility of the extended stacking faults. Consider that these are being formed as a result of dissociation of $a / 2\langle 110\rangle$ dislocations. Therefore, we anticipate that APBs must be created in the secondary precipitates if the extended stacking fault was to translate on the $\left\{\begin{array}{lll}1 & 1 & 1\end{array}\right\}$ plane. In fact, we do not observe APB contrast in the secondary precipitates under conditions where extended faulting is occurring (or any conditions in fact), due to the high energy associated with such large APB faults. In fact, Chen and Knowles [16] in their analysis of faults in CMSX-4 propose that only the 2D $\alpha$ partial is mobile, and that the $\mathrm{B} \alpha$ partial is sessile. However, if this were the case, only very limited strain levels could be achieved through the extension of the faults - far less than that required to explain the imposed strain levels. There is instead an alternative view regarding this mobility question. A second reordering step in the wake of the $\mathrm{B} \alpha$ composite partial could effectively eliminate the incorrect nearest neighbors that would otherwise be left on the primary $\left\{\begin{array}{lll}1 & 1 & 1\end{array}\right\}$ slip plane within the particles in the form of an APB. Assuming that reordering is complete, then a perfect crystal would instead be produced in both particle and matrix. This crucial insight immediately suggests that these extended 
faults may indeed be important, strain-producing elements in the substructure.

\subsection{Climb by-pass}

At the highest temperatures for which creep testing has been performed, a final mode of deformation appears - climb bypass of $a / 2\langle 110\rangle$ dislocations. We note that this mechanism has led to several well-established creep models for particle and precipitate strengthened alloys [17]. With respect to disk applications, however, this climb by-pass regime appears to be well-beyond the practical service temperatures. Its occurrence also seems to be correlated with the disappearance of the tertiary particle population due to Oswald ripening processes. The important role of the tertiary precipitates in potentially dictating some of these transitions will now be discussed.

\section{Discussion}

In this paper, we have reviewed the rich variety of mechanisms that appear to be operative as temperature is increased. At low temperatures, $a / 2\langle 110\rangle$ dislocation activity dominates, with planar deformation and shearing of particles by APB coupled dislocations. At temperatures in the range of $650-760^{\circ} \mathrm{C}$, isolated shearing of precipitates and the surprising appearance of microtwinning becomes dominant. While this is a narrow window of temperature, it is a crucial regime to fully understand in terms of enhancing the temperature and sustained peak load capabilities of these alloys. In this temperature range, microtwinning is operative under very low strain rate conditions, and appears to be the first plasticity process to become operative at lowest stress levels. Therefore, it is the process that controls the crucial lower bound for creep, and a microstructure based model for this regime has been reviewed. At slightly higher temperatures $\left(760^{\circ} \mathrm{C}\right)$ extended stacking faults are observed, often as extrinsic faults. These SESF-producing mechanisms are linked to the microtwinning process in that reordering in the secondary precipitates is required to remove the incorrect nearest neighbors that would otherwise be created in the wake of identical pairs of Shockley partials. It is therefore argued that these processes all require sufficient thermal activation to become active, and to supercede the pair-wise shearing process operative at lower temperatures.

These dramatic mechanism changes are also affected by the initial microstructure, or by changes in microstructure that also take place in this temperature regime. The clearest example of this sensitivity to microstructure is the appearance of isolated faulting in coarse microstructure and microtwinning in the fine microstructure. The fundamental difference between the two deformation modes is that for isolated faulting, $1 / 2\langle 110\rangle$ dislocations are operative in the matrix, while for microtwinning the leading dislocation is a $1 / 3\langle 112\rangle$ partial (which is comprised of two closely coupled $1 / 6\langle 112\rangle$ Shockley partials on adjacent $\left\{\begin{array}{llll}1 & 1 & 1\end{array}\right\}$ planes). Therefore, in the former mode, APBs must be created in the tertiary $\gamma^{\prime}$ particles, while in the latter mode, SESFs are created in the tertiary $\gamma^{\prime}$ (assuming that sufficient thermal activation exists for Kolbe reordering to occur).

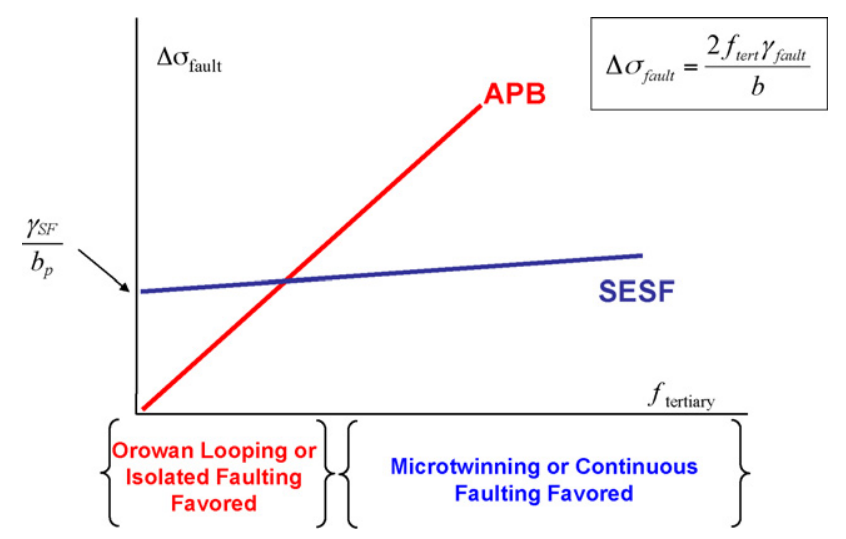

Fig. 7. Schematic depicting the strength required to shear $\gamma^{\prime}$ by APB or SESF mechanisms as a function of tertiary $\gamma^{\prime}$ volume fraction and conditions under which these deformation modes are favored.

To a first approximation, the additional stress required to shear a coherent particle varies as $2 f \gamma / b$, where $f$ is the particle volume fraction and $\gamma$ is the energy of the fault created in the particle [18]. For the two deformation modes in these alloys, the situation is schematically illustrated in Fig. 7. At small tertiary volume fraction, the APB mode of shearing is favored since this does not create a fault in the matrix, while for high volume fraction the SESF mode is favored since the energy of this fault is much lower. Thus, we can qualitatively rationalize a transition in deformation mode on this basis.

The tertiary $\gamma^{\prime}$ size and volume fraction coarsens over extended time at the creep temperatures, based on our microstructural observations. Therefore, creep conditions for which microtwinning is active initially may exhibit a gradual change to isolated faulting or Orowan looping if microstructural coarsening is sufficient during creep exposure. Another excellent example of the importance of the tertiary population is found in the recent work of Locq et al. [19] in which they show that preaging causes a significant increase in creep rate. This dramatic mechanical property change is due to a change in mechanism. In their case, they report a change from extended faulting in the unaged samples to climb by-pass in aged material. This transition is consistent with those that we have discussed related to the higher temperature regime. Note that this explanation for the effect of aging is quite different from that recently proposed by McLean and co-workers [20] in which they invoke the concept of an internal stress, and its relaxation during aging.

\section{Conclusions}

A surprising variety of apparently distinct deformation mechanism occur in polycrystalline Ni-base disk superalloys at temperatures above about $600-650^{\circ} \mathrm{C}$. The operative mechanisms depend on the scale of the microstructure (i.e. cooling rate), temperature and stress level. While a systematic study of these variations remains to be performed, the following general conclusions may be drawn. At lower temperatures in this regime, and at high stress, secondary particles are looped by $a / 2\langle 110\rangle$ dislocations, and then sheared by forming narrow APB ribbons between the loop and a trailing $a / 2\langle 110\rangle$ dislocation. At the 
highest temperatures explored in this study, $a / 2\langle 110\rangle$ dislocation by-pass the secondary particles via climb. At temperatures intermediate between these two extremes, $a / 2\langle 110\rangle$ activity is surprisingly replaced by shearing modes involving formation of continuous microtwins, as well as extended, fault configurations, frequently producing SESFs. It is proposed that these latter two processes are closely related: the propagation of the microtwins and extension of the SESFs occurs by the motion of identical $a / 6\langle 112\rangle$ Shockley partials, and that their movement is limited by a diffusion-mediated re-ordering which restores the $\mathrm{L}_{2}$ structure in the precipitates. There is also strong evidence indicating that the tertiary volume fraction is important in dictating these intermediate twinning and extended faulting modes. These results have revealed the possible thermally activated processes controlling creep in this temperature regime. This work also suggests that a complete, physically based model for creep will require multiple mechanisms to operate in parallel, and must also include the effect of evolving tertiary volume fraction during temperature exposure.

\section{Acknowledgements}

Support for this work has been provided by the DARPA Accelerated Insertion of Materials (AIM) Program under contract F33615-00-C-5215 and by the Air Force Office of Scientific Research for support of the model development through the MEANS-2 theme through Grant \# FA9550-05-1-0135.

\section{References}

[1] B.H. Kear, G.R. Leverant, J.M. Oblak, Trans. ASM 62 (1969) 639-650.

[2] J.P. Hirth, J. Lothe, Theory of Dislocations, 2nd ed., Wiley, New York, 1968, p. 319.

[3] M. Condat, B. Decamps, Scr. Metall. 21 (1987) 607-612.

[4] B. Decamps, A.J. Morton, M. Condat, Phil. Mag. A 64 (1991) 641-668.

[5] B. Decamps, S. Raujol, A. Coujou, F. Pettinari-Sturmel, N. Clement, D. Locq, P. Caron, Phil. Mag. A 84 (2004) 91-104.

[6] B. Decamps, A.J. Morton, Phil. Mag. A 68 (1993) 1129-1149.

[7] D.M. Knowles, Q.Z. Chen, Mater. Sci. Eng. A 340 (2003) 88-102.

[8] M. Kolbe, Mater. Sci. Eng. A 319-321 (2001) 383-387.

[9] M. Kolbe, J. Pelissier, M. Ignat, B. Shollock, M. McLean, M. Dupeux, A. Kalogeridis, R. Maldonado, E. Nembach, Proceedings of ICSMA 10, The Japan Institute of Metals, Sendai, 1994, p. 685.

[10] G.B. Viswanathan, P.M. Sarosi, M.F. Henry, D.D. Whitis, W.W. Milligan, M.J. Mills, Acta Mater. 53 (2005) 3041-3057.

[11] P.M. Sarosi, G.B. Viswanathan, M.J. Mills, Scr. Mater. 55 (2006) 727-730.

[12] S. Karthikeyan, R.R. Unocic, P.M. Sarosi, G.B. Viswanathan, D.D. Whitis, M.J. Mills, Scr. Mater. 54 (2006) 1157-1162.

[13] S. Karthikeyan, unpublished research.

[14] R.R. Unocic, M.J. Mills, unpublished research.

[15] B. Reppich, P. Schepp, G. Wehner, Acta Metall. 30 (1982) 95-104.

[16] Q.Z. Chen, D.M. Knowles, Mater. Sci. Eng. A 356 (2003) 352-367.

[17] A. Manonukul, F.P.E. Dunne, D. Knowles, Acta Mater. 50 (2002) 2917-2931.

[18] E. Nembach, G. Neite, Prog. Mater. Sci. 29 (1985) 177-319.

[19] D. Locq, P. Caron, S. Raujol, F. Pettinari-Sturmel, A. Coujou, N. Clement, Proceedings of the 10th International Symposium on Superalloys, 2004, pp. 179-187.

[20] S.K. Sondhi, B.F. Dyson, M. McLean, Acta Mater. 52 (2004) 17611772. 